Review

\title{
Advances in Nutritional Research on Regulatory T-Cells
}

\section{Wooki Kim ${ }^{1}$ and Hyungjae Lee ${ }^{2, *}$}

1 Department of Food Science and Biotechnology, Graduate School of Biotechnology, Kyung Hee University, Yongin, Gyeonggi 446-701, Korea; E-Mail: kimw@khu.ac.kr

* Author to whom correspondence should be addressed; E-Mail: lee252@dankook.ac.kr; Tel.: +82-41-550-3561; Fax: +82-41-559-7868.

Received: 29 July 2013; in revised form: 17 October 2013 / Accepted: 22 October 2013 / Published: 28 October 2013

\begin{abstract}
Many clinical and animal studies have shown that certain dietary components exert anti-inflammatory properties that aid in the amelioration of chronic inflammatory diseases. Among the various proposed channels through which dietary components affect immune responses, regulatory T-cells (Tregs) are emerging as key targets for the dietary prevention of chronic inflammatory diseases. In this review, immunoregulation by Tregs is briefly described, followed by a summary of recent advances and possible applications of techniques for the study of Tregs. In addition, this review provides an overview of the current knowledge on Treg regulation by certain dietary components, including vitamins, omega-3 polyunsaturated fatty acids, and polyphenols. The caveats of previous studies are also discussed in order to highlight the distinctions between dietary studies and immunological approaches. Consequently, this review may help to clarify the means by which nutritional components influence Tregs.
\end{abstract}

Keywords: nutrition; immunology; regulatory T-cells; Tregs; anti-inflammatory

\section{Introduction to T-Cell Immunology}

Higher organisms, including humans, have immune systems developed to protect the host from potentially harmful materials originating both endogenously and exogenously. In other words, immune responses can also be described as a mechanism of distinguishing "self" from "non-self" or "abnormal self". One of a variety of immunological constituents, T-cells play a pivotal role in both innate and 
adaptive immune responses [1], directly by exerting cytotoxic effects on infected cells or indirectly by modulating other types of immune cells. Cytotoxic T-cells (Tc), also called cluster of differentiation (CD) $8^{+}$T-cells due to their characteristic expression of CD8 protein on cell surfaces, mediate the direct toxicity against infected cells.

Indirect immunomodulation is controlled by $\mathrm{CD}^{+}{ }^{+}$-cells, also called helper T-cells (Th), which regulate other immune cell types. During activation by antigen encounter, naïve (previously antigen-unexposed) $\mathrm{CD}^{+}{ }^{+} \mathrm{T}$-cells (Th0) are further differentiated into effector subtypes, which have been distinguished to date as Th1, Th2, Th9, Th17, and Th22 cells, depending on the milieu of tissues, type of antigen, and corresponding co-stimulating molecules on the antigen-presenting cells. The Th1 subtype, distinguished by nuclear factors STAT1, STAT4, and T-bet, produces interferon (IFN)- $\gamma$, tumor necrosis factor (TNF)- $\alpha$, interleukin(IL)-2, and IL-12, resulting in the activation of effector cytotoxic $\mathrm{CD}^{+} \mathrm{T}$-cells and innate immune cells such as macrophages [2,3]. Th2 cells, in which the nuclear factors STAT6 and GATA3 are present, secrete IL-4, IL-5, IL-10, and IL-13, which further activate B-cell transformation to plasma cells in order to provide humoral immunity through the production of antibodies. In contrast to the aforementioned systemic helper T-cells, IL-17-producing Th17 cells have been identified in local inflammation-prone sites, including the digestive tract. It has been reported that the orphan retinoic acid receptor (ROR) family transcription factor ROR $\gamma \mathrm{t}$ is essential for Th17 development and function [4], indicating that vitamin A, a precursor of retinoic acid, is a potential dietary modulator of Th17 cells and consequent inflammatory responses. Indeed, numerous data have indicated that Th17 cells are highly relevant to the onset and propagation of local chronic inflammation, in part by producing inflammatory cytokines, including IL-17 [5-8]. IL-22-producing Th22 cells have been demonstrated to be central to host protection against bacterial infections at barrier sites. The development of Th22 cells is dependent on the expression of the transcription factors T-bet and AhR [9]. Th9 cells were identified by the production of IL-9 [10,11], the in vivo significance of which has not been clearly identified, although Th9 cells are thought to be involved in allergic inflammation, anti-tumor immunity, and autoimmune inflammation (reviewed in [12]). The differentiation of $\mathrm{CD}^{+}{ }^{+}$-cells is summarized in Figure 1.

Figure 1. Schematic representation of $\mathrm{CD} 4{ }^{+} \mathrm{T}$-cell differentiation and characteristic cytokine production of differentiated subtypes. GALT stands for gut-associated lymphoid tissue.

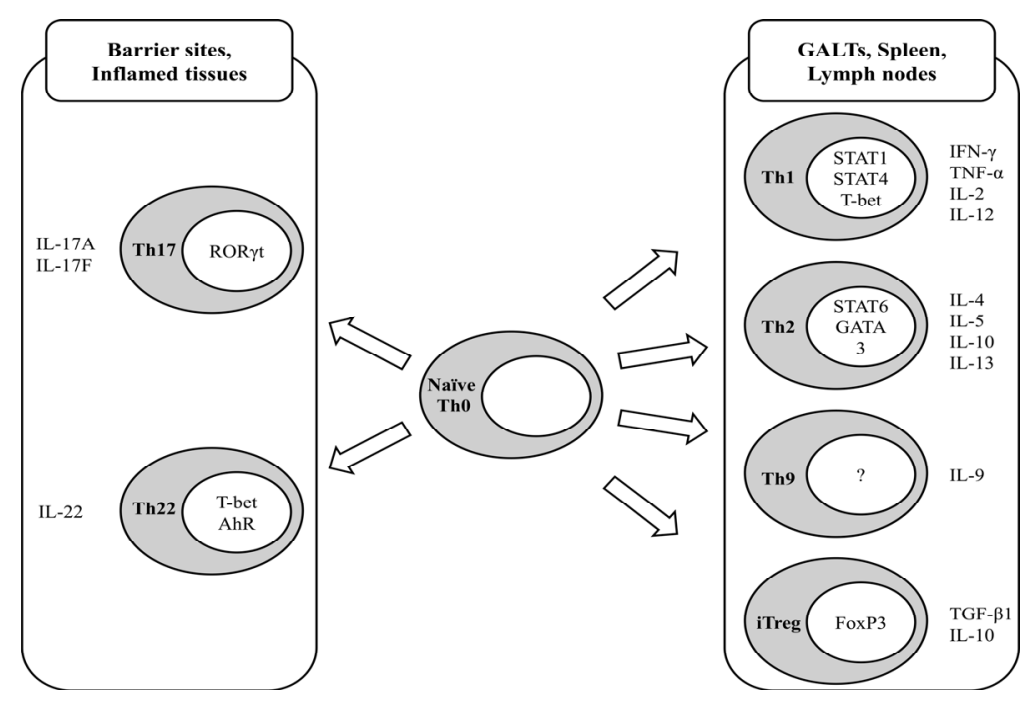




\section{Regulatory T-Cells}

In contrast to the aforementioned T-cell subtypes, all of which promote specific types of immune responses, regulatory T-cells (Tregs) were identified in the late 1970s by their unique ability to suppress immune responses [13]. Two distinctive regulatory mechanisms of Tregs have been discovered: direct cell-cell contact with inflammatory effector T-cells and secretion of anti-inflammatory cytokines, such as TNF- $\alpha$ and IL-10 [14]. As for the development of the cell lineage, Tregs develop in either the thymus (natural Tregs, nTregs) or secondary lymphoid tissues (inducible Tregs, iTregs) such as spleen, lymph nodes, and intestinal mucosa [15]. nTregs require CD28 costimulation during positive selection in the thymus in the presence of TGF- $\beta$, IL-2, and IL-15. It has been reported that Helios, an Ikaros transcription factor family member, is uniquely expressed in nTregs [16] but not in iTregs. However, this is still under debate owing to the recent report of the coexistence of both Helios ${ }^{+}$and Helios nTregs in vivo [17]. Because of their low prevalence among $\mathrm{CD}^{+} \mathrm{T}$-cells in vivo, the function of nTregs is not clear at present, but they are thought to be responsible for the suppression of inflammatory signals, either by cell-cell contact, secretion of the anti-inflammatory cytokines TGF- $\beta 1$ and IL-10, or both. iTregs, however, develop under subinflammatory conditions, such as in inflamed tissues or normal intestinal mucosa with costimulation of CTLA-4 in the presence of TGF- $\beta$ and IL- 2 . iTregs exert anti-inflammatory effects on activated effector T cells through the secretion of IL-10 and TGF- $\beta 1$. Because iTregs develop from naïve $\mathrm{CD} 4^{+} \mathrm{T}$-cells in the periphery lymphoid tissues, the repertoire of iTregs is specific to allergens, commensal microbiota, neoantigens, alloantigens, and self-antigens [18,19].

Through the use of immunostaining techniques with specific antibodies, iTregs were found to express CD25 molecules, a surface marker of "antigen experienced" T-cells, indicating that iTregs are not naïve but develop through certain inflammatory responses. In fact, iTregs are specifically abundant in the digestive tract, respiratory tract, and other inflammatory sites, where the influx of exogenous materials is a common occurrence. It is well known that a variety of microbiota living in the intestinal tract discharge a tremendous amount of microbial products such as lipopolysaccharides, flagellins, double-stranded RNAs, and DNA fragments rich in cytosine and guanine (CpG islands). Regulation of the immune system to properly maintain host defenses while suppressing chronic inflammatory responses to remarkable amounts of bacterial products is not yet completely understood. Recently, iTregs were recognized as one of the major constituents that regulate intestinal inflammatory disease [20,21]. Furthermore, in a mouse model with increased gut permeability resulting from the genetic deletion of Junctional Adhesion Molecule-A (JAM-A), an increased influx of microbial products provoked immune responses, resulting in the development of inflammatory Th17 cells as well as anti-inflammatory iTregs [22]. Thus, an analogy is frequently drawn between iTregs and "firemen" in "burning" (i.e., inflamed) sites.

\section{In vitro Induction and Intracellular Staining of iTregs}

As mentioned above, iTregs are unique in that they develop from the same progenitor cells as inflammatory effector T-cells. Therefore, the increasing focus on iTregs as targets for regulation of inflammation by therapeutic or nutritional means requires experimental approaches to identify iTregs 
in a mixed population of cells. The differentiation of $\mathrm{CD}^{+} \mathrm{T}$-cells into a specific subpopulation is dependent on the expression of nuclear factors. The nuclear factor forkhead box P3 (Foxp3) was found to be such a key constituent, and directs the conversion of naïve $\mathrm{CD} 4^{+} \mathrm{T}$-cells into Tregs [23].

An advance in immunostaining techniques enabled the staining of intracellular nuclear factors, including Foxp3; briefly, a single-cell suspension of a cell mixture is stained using monoclonal antibodies specific to CD4 and T-cell receptor $\beta$ (TCR $\beta$ ), followed by cell fixation and permeabilization. After washing, a monoclonal antibody specific to Foxp3 is applied in order to stain that nuclear factor. The stained cells are analyzed by flow cytometry, followed by analysis using dedicated software. A typical characterization of Tregs by immunostaining and flow cytometric analysis of a single-cell suspension derived from murine colon is shown in Figure 2.

Figure 2. Flow cytometric analysis of Tregs by intracellular staining of Foxp3. (A) Lymphocytes are gated by forward scatter (FSC) vs. side scatter (SSC), followed by (B) $\mathrm{CD}^{+}$T-cell gating on CD4 and TCR $\beta$. (C) Foxp3 ${ }^{+}$Tregs gated out of $\mathrm{CD} 4^{+} \mathrm{TCR}^{+}$ T-cells. The numbers indicate the percentage of the gated cells out of the total number of cells within the plot.

(A)

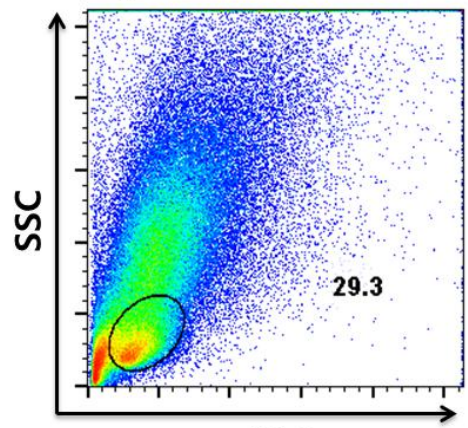

FSC
(B)

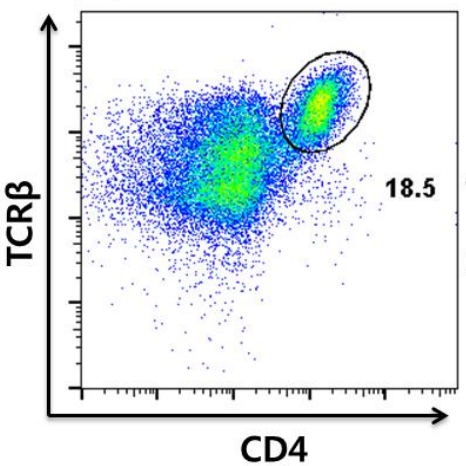

(C)

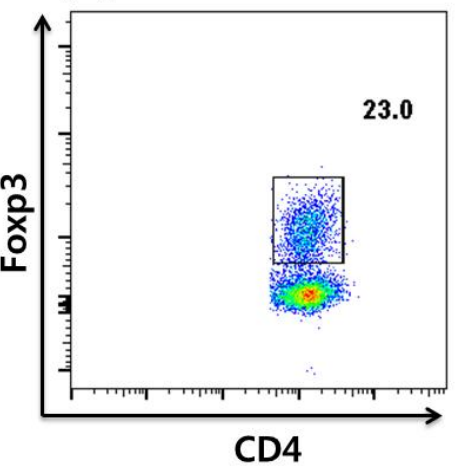

As for experiments testing the direct role of specific factors in Treg differentiation from naïve T-cells, in vivo studies are complicated by the fact that various cells interact with each other. Ex vivo differentiation of naïve $\mathrm{CD}^{+}$T-cells into Tregs (Ti-Treg) was made feasible by culture of the naïve cells in the presence of Treg-promoting cytokines [24]. Briefly, naïve CD4 ${ }^{+} \mathrm{CD} 25^{-} \mathrm{T}$-cells are purified using surface marker-specific magnetic beads and magnetic separators, followed by culture with mitogenic antibodies (anti-CD3 and anti-CD28 monoclonal antibodies) and transforming growth factor (TGF)- $\beta 1$. By following this protocol, more than $60 \%$ of $\mathrm{CD}^{+}$T-cells were converted to Foxp $3^{+}$ Ti-Tregs in five days [24]. These ex vivo converted Ti-Tregs were further shown to express Foxp3 mRNA and to properly suppress effector T-cell proliferation, as assessed by real-time PCR and an intracellular dye (CFSE) dilution test, respectively. Therefore, this method of induction of Ti-Tregs may be a potent tool to investigate the direct role of specific environmental factors, including food ingredients, in the differentiation of Tregs. 


\section{Dietary Regulation of Tregs}

\subsection{Vitamins}

Following dietary consumption and absorption in the intestines, water-insoluble vitamin A (all-trans-retinol) is carried by cellular retinol binding protein (CRBP) in an aqueous environment to be transported into the cytoplasm. Subsequently, retinol is oxidized to retinal by retinol dehydrogenase, and then retinal is further oxidized to retinoic acid by retinal dehydrogenase [25]. With respect to the role of vitamin A in the development of Tregs, Bai et al. [26] demonstrated that the population of Tregs derived from biopsies of ulcerative colitis patients increases following ex vivo culture in the presence of retinoic acid, a potent metabolite of vitamin A. In the same study, using a chemical (2,4,6-trinitrobenzene sulfonic acid, TNBS)-induced murine colitis model, it was further elucidated that dietary vitamin A ameliorated colitis, which is accompanied by an increased population of Tregs. Wu et al. [27] further reported that intraperitoneal administration of all-trans retinoic acid aided in the attenuation of airway inflammation by inducing Treg development in a model of experimental allergic asthma. Moreover, a dietary study demonstrated that retinal intervention in mice upregulated Tregs, which further assisted in the treatment of autoimmune inflammatory disorders, including rheumatoid arthritis [28]. Overall, these animal studies indicate that vitamin A and its metabolites affect populations of Tregs, thereby suppressing chronic inflammatory diseases. However, it remains unclear whether vitamin A affects Tregs directly, indirectly, or both. Indeed, Chang et al. demonstrated that dendritic cells also promote the generation of Tregs in response to retinoic acid, at least in vitro [29], exemplifying that in vivo models are more complicated for elucidating the mechanism of action of dietary components.

With respect to water-soluble vitamins, Kunisawa et al. [30] showed that Tregs express high levels of vitamin $\mathrm{B}_{9}$ (folic acid) receptor on their cell surfaces. Furthermore, vitamin $\mathrm{B}_{9}$ was demonstrated to be a survival factor for Tregs; in a vitamin $\mathrm{B}_{9}$-deficient culture, naïve $\mathrm{CD}^{+} \mathrm{T}$-cells successfully differentiated into Foxp $3^{+}$Tregs but failed to survive. Moreover, it was found that mice fed a vitamin $\mathrm{B}_{9}$-deficient diet exhibited a decreased number of Tregs in the small intestine, where vitamin $\mathrm{B}_{9}$ is absorbed.

\subsection{Dietary Fatty Acids}

A significant quantity of data has indicated that dietary omega-3 polyunsaturated fatty acids (PUFA) may prevent or ameliorate chronic inflammatory diseases, including inflammatory bowel diseases [31-34]. These studies have identified multiple anti-inflammatory mechanisms of omega-3 PUFA: cytokine production, antagonism to omega-6 PUFA metabolism, binding to nuclear receptors as ligands, the alteration of signaling protein acylation, and the modulation of signaling platform lipid rafts in various immune cell models. With respect to $\mathrm{CD} 4^{+} \mathrm{T}$-cell mediated inflammatory responses, several studies using dietary intervention with either purified omega-3 PUFA or fish oil (which is rich in omega-3 PUFA) demonstrated that $\mathrm{CD}^{+}$T-cell functions, as assessed by cytokine production and proliferation, were suppressed in both humans [35-37] and experimental animals [38,39].

As for the effect of omega-3 PUFA on Tregs, it was shown that the omega-3 PUFA abundant in fermented fish oil enhanced the development of Foxp $3^{+}$Tregs in vivo [40]. Moreover, intraperitoneal 
injection of eicosapentaenoic acid (EPA), an omega-3 PUFA, resulted in prolongation of graft survival in a murine transplant model, accompanied by an increased population of Tregs [41]. However, those studies do not conclusively demonstrate a direct function of omega-3 PUFA on the differentiation and/or function of Tregs, given the caveat that in vivo administration of omega-3 PUFA can affect diverse types of accessory cells. Indeed, it was found that dendritic cells cultured in docosahexaenoic acid (DHA, an omega-3 PUFA)-rich conditions facilitated the development of Tregs, at least in vitro [42], indicating an indirect modulation of Treg development by omega-3 PUFA. However, despite the increase in the development of Tregs, the favorable effect of omega-3 PUFA on Tregs is still controversial because it has also been observed that DHA inhibited the suppressive effect of Tregs on effector T-cell proliferations in a dose-dependent manner in vitro [43].

\subsection{Dietary Polyphenols}

Dietary polyphenols are well known for their antioxidant properties, which can further suppress inflammatory responses by reducing nitric oxide. In addition to their archetypal antioxidant capacities, immunoregulatory effects of select polyphenols on Tregs have been characterized as follows. Wang et al. [44] reported that ex vivo development of $\mathrm{CD}^{+}{ }^{+}$Foxp $3^{+}$Tregs was induced in the presence of the flavonoid naringenin in part via activation of the aryl hydrocarbon receptor, a transcription factor. As well, increased Treg differentiation suppressed the proliferation of effector T-cells. In addition, dietary naringenin was found to suppress cholesterol-induced systemic inflammation, metabolic dysregulation, atherosclerosis, and allergen-induced airway inflammation [45,46], although it was not clear whether those inhibitory effects were Treg-dependent. In a mouse model of T-cell mediated inflammatory disease of the central nervous system, dietary intake of epigallocatechin gallate (EGCG), a catechin derivative, exerted a favorable effect, in part by increasing the Treg population in the spinal cord [47]. Furthermore, fermented grape marc (FGM) was found to promote Treg differentiation of human CD $4^{+}$ T-cells [48].

\section{Conclusions}

Due to the relatively short history of studies on Tregs, investigations of the direct effects of a variety of dietary components on Treg conversion are limited at present (summarized in Table 1 and previously reviewed elsewhere [49]). It seems that a majority of the anti-inflammatory functional studies have been conducted in vivo by observing physiological outcomes, such as symptoms of chronic inflammation and accumulation of certain immune cell types. Even though in vivo studies are indispensable for nutritional studies, simpler model systems must be developed to dissect the detailed mechanisms by which dietary components regulate physiological systems. Therefore, further studies using more sophisticated and appropriate in vivo model systems are needed to draw solid conclusions. In this regard, as introduced in this review, the protocol for Treg differentiation from naïve $\mathrm{CD}^{+}{ }^{+} \mathrm{T}$ cells could be effectively utilized to determine the direct effects of a variety of promising dietary Treg modulators present in foods. 
Table 1. Summary of the modulation of Tregs by dietary components.

\begin{tabular}{|c|c|c|}
\hline $\begin{array}{c}\text { Dietary } \\
\text { component }\end{array}$ & Function & Reference \\
\hline Vitamin A & $\begin{array}{c}\text { Increased Treg population in human biopsy culture from } \\
\text { ulcerative colitis patients in vitro. } \\
\text { Amelioration of TNBS-induced murine } \\
\text { colitis model in vivo. }\end{array}$ & {$[26]$} \\
\hline Vitamin $\mathbf{B}_{9}$ & $\begin{array}{l}\text { Essential for murine Treg survival ex vivo. } \\
\text { Increased Treg population in murine } \\
\text { small intestine in vivo. }\end{array}$ & {$[30]$} \\
\hline & Enhanced development of murine Tregs in vivo. & [37] \\
\hline Omega-3 PUFA & $\begin{array}{l}\text { Increased Tregs in a murine transplant model in vivo. } \\
\text { Increased ability of murine dendritic cells to } \\
\text { convert Tregs in vitro. }\end{array}$ & $\begin{array}{l}{[38]} \\
{[39]}\end{array}$ \\
\hline & Decreased regulatory function of murine Tregs ex vivo. & [40] \\
\hline \multicolumn{3}{|l|}{ Polyphenols } \\
\hline Naringenin & Increased murine Treg population in vivo. & [44] \\
\hline $\mathrm{EGCG}^{1}$ & Increased murine Treg population in vivo. & [47] \\
\hline $\mathrm{FGM}^{2}$ & Increased human Treg population in vivo. & [48] \\
\hline
\end{tabular}

\section{Acknowledgments}

This research was supported by the Basic Science Research Program through the National Research Foundation of Korea funded by the Ministry of Science, ICT and Future Planning (NRF2013R1A1A1006288) and by a grant from Kyung Hee University in 2012 (KHU-20120570).

\section{Conflicts of Interest}

The authors declare no conflict of interest.

\section{References}

1. Jager, A.; Kuchroo, V.K. Effector and regulatory T-cell subsets in autoimmunity and tissue inflammation. Scand. J. Immunol. 2010, 72, 173-184.

2. Stout, R.D.; Bottomly, K. Antigen-specific activation of effector macrophages by IFN-gamma producing (TH1) T cell clones. Failure of IL-4-producing (TH2) T cell clones to activate effector function in macrophages. J. Immunol. 1989, 142, 760-765.

3. Heinzel, F.P.; Sadick, M.D.; Mutha, S.S.; Locksley, R.M. Production of interferon gamma, interleukin 2, interleukin 4, and interleukin 10 by CD4+ lymphocytes in vivo during healing and progressive murine leishmaniasis. Proc. Natl. Acad. Sci. USA 1991, 88, 7011-7015.

4. Chen, Z.; Lin, F.; Gao, Y.; Li, Z.; Zhang, J.; Xing, Y.; Deng, Z.; Yao, Z.; Tsun, A.; Li, B. FOXP3 and RORgammat: Transcriptional regulation of Treg and Th17. Int. Immunopharmacol. 2011, 11, 536-542. 
5. Chang, Q.; Wang, Y.K.; Zhao, Q.; Wang, C.Z.; Hu, Y.Z.; Wu, B.Y. Th17 cells are increased with severity of liver inflammation in patients with chronic hepatitis C. J. Gastroenterol. Hepatol. 2012, 27, 273-278.

6. Murdaca, G.; Colombo, B.M.; Puppo, F. The role of Th17 lymphocytes in the autoimmune and chronic inflammatory diseases. Intern. Emerg. Med. 2011, 6, 487-495.

7. Caprioli, F.; Pallone, F.; Monteleone, G. Th17 immune response in IBD: A new pathogenic mechanism. J. Crohn's Colitis 2008, 2, 291-295.

8. Seiderer, J.; Elben, I.; Diegelmann, J.; Glas, J.; Stallhofer, J.; Tillack, C.; Pfennig, S.; Jurgens, M.; Schmechel, S.; Konrad, A.; et al. Role of the novel Th17 cytokine IL-17F in inflammatory bowel disease (IBD): Upregulated colonic IL-17F expression in active Crohn's disease and analysis of the IL17F p.His161Arg polymorphism in IBD. Inflamm. Bowel Dis. 2008, 14, 437-445.

9. Basu, R.; O’Quinn, D.B.; Silberger, D.J.; Schoeb, T.R.; Fouser, L.; Ouyang, W.; Hatton, R.D.; Weaver, C.T. Th22 cells are an important source of IL-22 for host protection against enteropathogenic bacteria. Immunity 2012, 37, 1061-1075.

10. Dardalhon, V.; Awasthi, A.; Kwon, H.; Galileos, G.; Gao, W.; Sobel, R.A.; Mitsdoerffer, M.; Strom, T.B.; Elyaman, W.; Ho, I.C.; et al. IL-4 inhibits TGF-beta-induced Foxp3+ T cells and, together with TGF-beta, generates IL-9+ IL-10+ Foxp3(-) effector T cells. Nat. Immunol. 2008, 9, 1347-1355.

11. Veldhoen, M.; Uyttenhove, C.; van Snick, J.; Helmby, H.; Westendorf, A.; Buer, J.; Martin, B.; Wilhelm, C.; Stockinger, B. Transforming growth factor-beta 'reprograms' the differentiation of T helper 2 cells and promotes an interleukin 9-producing subset. Nat. Immunol. 2008, 9, 1341-1346.

12. Kaplan, M.H. Th9 cells: Differentiation and disease. Immunol. Rev. 2013, 252, 104-115.

13. Baker, P.J. Homeostatic control of antibody responses: A model based on the recognition of cell-associated antibody by regulatory T cells. Transplant. Rev. 1975, 26, 3-20.

14. Baumgart, M.; Tompkins, F.; Leng, J.; Hesse, M. Naturally occurring CD4+Foxp3+ regulatory T cells are an essential, IL-10-independent part of the immunoregulatory network in Schistosoma mansoni egg-induced inflammation. J. Immunol. 2006, 176, 5374-5387.

15. Yuan, X.; Malek, T.R. Cellular and molecular determinants for the development of natural and induced regulatory T cells. Hum. Immunol. 2012, 73, 773-782.

16. Thornton, A.M.; Korty, P.E.; Tran, D.Q.; Wohlfert, E.A.; Murray, P.E.; Belkaid, Y.; Shevach, E.M. Expression of Helios, an Ikaros transcription factor family member, differentiates thymic-derived from peripherally induced Foxp3+ T regulatory cells. J. Immunol. 2010, 184, 3433-3441.

17. Himmel, M.E.; MacDonald, K.G.; Garcia, R.V.; Steiner, T.S.; Levings, M.K. Helios+ and Helios - cells coexist within the natural FOXP3+ T regulatory cell subset in humans. J. Immunol. 2013, 190, 2001-2008.

18. Curotto de Lafaille, M.A.; Lafaille, J.J. Natural and adaptive foxp3+ regulatory T cells: More of the same or a division of labor? Immunity 2009, 30, 626-635.

19. Toda, A.; Piccirillo, C.A. Development and function of naturally occurring CD4+CD25+ regulatory T cells. J. Leukoc. Biol. 2006, 80, 458-470.

20. Tanoue, T.; Honda, K. Induction of Treg cells in the mouse colonic mucosa: A central mechanism to maintain host-microbiota homeostasis. Semin. Immunol. 2012, 24, 50-57. 
21. Kurzweil, V.; Tarangelo, A.; Oliver, P.M. Gastrointestinal microbiota do not significantly contribute to T cell activation or GI inflammation in Ndfip1-cKO mice. PLoS One 2012, 7, e34478.

22. Khounlotham, M.; Kim, W.; Peatman, E.; Nava, P.; Medina-Contreras, O.; Addis, C.; Koch, S.; Fournier, B.; Nusrat, A.; Denning, T.L.; et al. Compromised intestinal epithelial barrier induces adaptive immune compensation that protects from colitis. Immunity 2012, 37, 563-573.

23. Koch, U.; Radtke, F. Mechanisms of T cell development and transformation. Annu. Rev. Cell Dev. Biol. 2011, 27, 539-562.

24. Fantini, M.C.; Dominitzki, S.; Rizzo, A.; Neurath, M.F.; Becker, C. In vitro generation of CD4+ CD25+ regulatory cells from murine naive T cells. Nat. Protoc. 2007, 2, 1789-1794.

25. Maden, M. Retinoid signalling in the development of the central nervous system. Nat. Rev. Neurosci. 2002, 3, 843-853.

26. Bai, A.; Lu, N.; Guo, Y.; Liu, Z.; Chen, J.; Peng, Z. All-trans retinoic acid down-regulates inflammatory responses by shifting the Treg/Th17 profile in human ulcerative and murine colitis. J. Leukoc. Biol. 2009, 86, 959-969.

27. Wu, J.; Zhang, Y.; Liu, Q.; Zhong, W.; Xia, Z. All-trans retinoic acid attenuates airway inflammation by inhibiting Th2 and Th17 response in experimental allergic asthma. BMC Immunol. 2013, 14, 28.

28. Park, M.K.; Jhun, J.Y.; Lee, S.Y.; Oh, H.J.; Park, M.J.; Byun, J.K.; Yoon, B.Y.; Park, E.M.; Lee, D.G.; Kwok, S.K.; et al. Retinal attenuates inflammatory arthritis by reciprocal regulation of IL-17-producing T cells and Foxp3(+) regulatory T cells and the inhibition of osteoclastogenesis. Immunol. Lett. 2012, 148, 59-68.

29. Chang, J.; Thangamani, S.; Kim, M.H.; Ulrich, B.; Morris, S.M., Jr.; Kim, C.H. Retinoic acid promotes the development of Arg1-expressing dendritic cells for the regulation of T-cell differentiation. Eur. J. Immunol. 2013, 43, 967-978.

30. Kunisawa, J.; Hashimoto, E.; Ishikawa, I.; Kiyono, H. A pivotal role of vitamin B9 in the maintenance of regulatory T cells in vitro and in vivo. PLoS One 2012, 7, e32094.

31. Calder, P.C. Omega-3 polyunsaturated fatty acids and inflammatory processes: Nutrition or pharmacology? Br. J. Clin. Pharmacol. 2012, 75, 645-662.

32. Cabre, E.; Manosa, M.; Gassull, M.A. Omega-3 fatty acids and inflammatory bowel diseases-A systematic review. Br. J. Nutr. 2012, 107 (Suppl. 2), 240-252.

33. Maroufyan, E.; Kasim, A.; Ebrahimi, M.; Loh, T.C.; Bejo, M.H.; Zerihun, H.; Hosseni, F.; Goh, Y.M.; Farjam, A.S. Omega-3 polyunsaturated fatty acids enrichment alters performance and immune response in infectious bursal disease challenged broilers. Lipids Health Dis. 2012, 11, 15.

34. Wall, R.; Ross, R.P.; Fitzgerald, G.F.; Stanton, C. Fatty acids from fish: The anti-inflammatory potential of long-chain omega-3 fatty acids. Nutr. Rev. 2010, 68, 280-289.

35. Sorokovoi, K.V.; Pogozheva, A.V.; Sergeeva, K.V. Effect of diet including omega-3 polyunsaturated fatty acids on the status of T-cell immunity in patients with ischemic heart disease and hyperproteinemia. Vopr. Pitan. 1997, 5, 6-8.

36. Terada, S.; Takizawa, M.; Yamamoto, S.; Ezaki, O.; Itakura, H.; Akagawa, K.S. Suppressive mechanisms of EPA on human T cell proliferation. Microbiol. Immunol. 2001, 45, 473-481. 
37. Molvig, J.; Pociot, F.; Worsaae, H.; Wogensen, L.D.; Baek, L.; Christensen, P.; Mandrup-Poulsen, T.; Andersen, K.; Madsen, P.; Dyerberg, J.; et al. Dietary supplementation with omega-3-polyunsaturated fatty acids decreases mononuclear cell proliferation and interleukin-1 beta content but not monokine secretion in healthy and insulin-dependent diabetic individuals. Scand. J. Immunol. 1991, 34, 399-410.

38. Simopoulos, A.P. Omega-3 fatty acids in health and disease and in growth and development. Am. J. Clin. Nutr. 1991, 54, 438-463.

39. Curtis, C.L.; Rees, S.G.; Little, C.B.; Flannery, C.R.; Hughes, C.E.; Wilson, C.; Dent, C.M.; Otterness, I.G.; Harwood, J.L.; Caterson, B. Pathologic indicators of degradation and inflammation in human osteoarthritic cartilage are abrogated by exposure to $n-3$ fatty acids. Arthritis Rheum. 2002, 46, 1544-1553.

40. Han, S.C.; Kang, G.J.; Ko, Y.J.; Kang, H.K.; Moon, S.W.; Ann, Y.S.; Yoo, E.S. Fermented fish oil suppresses $\mathrm{T}$ helper $1 / 2$ cell response in a mouse model of atopic dermatitis via generation of CD4+CD25+Foxp3+ T cells. BMC Immunol. 2012, 13, 44.

41. Iwami, D.; Nonomura, K.; Shirasugi, N.; Niimi, M. Immunomodulatory effects of eicosapentaenoic acid through induction of regulatory T cells. Int. Immunopharmacol. 2011, 11, 384-389.

42. Kong, W.; Yen, J.H.; Ganea, D. Docosahexaenoic acid prevents dendritic cell maturation, inhibits antigen-specific Th1/Th17 differentiation and suppresses experimental autoimmune encephalomyelitis. Brain Behav. Immun. 2011, 25, 872-882.

43. Yessoufou, A.; Ple, A.; Moutairou, K.; Hichami, A.; Khan, N.A. Docosahexaenoic acid reduces suppressive and migratory functions of CD4+CD25+ regulatory T-cells. J. Lipid Res. 2009, 50, 2377-2388.

44. Wang, H.K.; Yeh, C.H.; Iwamoto, T.; Satsu, H.; Shimizu, M.; Totsuka, M. Dietary flavonoid naringenin induces regulatory $\mathrm{T}$ cells via an aryl hydrocarbon receptor mediated pathway. J. Agric. Food Chem. 2012, 60, 2171-2178.

45. Assini, J.M.; Mulvihill, E.E.; Sutherland, B.G.; Telford, D.E.; Sawyez, C.G.; Felder, S.L.; Chhoker, S.S.; Edwards, J.Y.; Gros, R.; Huff, M.W. Naringenin prevents cholesterol-induced systemic inflammation, metabolic dysregulation and atherosclerosis in $\mathrm{Ldll}^{-/-}$mice. J. Lipid Res. 2012, 54, 711-724.

46. Shi, Y.; Dai, J.; Liu, H.; Li, R.R.; Sun, P.L.; Du, Q.; Pang, L.L.; Chen, Z.; Yin, K.S. Naringenin inhibits allergen-induced airway inflammation and airway responsiveness and inhibits NF-kappaB activity in a murine model of asthma. Can. J. Physiol. Pharmacol. 2009, 87, 729-735.

47. Wang, J.; Ren, Z.; Xu, Y.; Xiao, S.; Meydani, S.N.; Wu, D. Epigallocatechin-3-gallate ameliorates experimental autoimmune encephalomyelitis by altering balance among CD4+ T-cell subsets. Am. J. Pathol. 2012, 180, 221-234.

48. Magrone, T.; Jirillo, E. Influence of polyphenols on allergic immune reactions: Mechanisms of action. Proc. Nutr. Soc. 2012, 71, 316-321. 
49. Issazadeh-Navikas, S.; Teimer, R.; Bockermann, R. Influence of dietary components on regulatory T cells. Mol. Med. 2012, 18, 95-110.

(C) 2013 by the authors; licensee MDPI, Basel, Switzerland. This article is an open access article distributed under the terms and conditions of the Creative Commons Attribution license (http://creativecommons.org/licenses/by/3.0/). 